\title{
Negotiation and take-it or leave-it in Common Agency with non-contractible actions
}

\author{
Andrea ATTAR $^{1}$, Gwenaël PIASER ${ }^{2}$ and Nicolás PORTEIRO ${ }^{3}$
}

January 2006

\begin{abstract}
This note presents a counter-example to Theorems 3 and 4 in Peters [3].

Keywords: Menus, Common Agency.

JEL Classification: D82.
\end{abstract}

\footnotetext{
${ }^{1}$ IDEI, Université de Toulouse I and Università di Roma, La Sapienza.

${ }^{2}$ Università Ca' Foscari di Venezia.

${ }^{3}$ Dpto. Economía M. Cuant. e Hํㅡㄹ Ec. Universidad Pablo de Olavide.
}

This note is an outcome of researches started when all the authors were working at CORE. Thus, we would like to thank all CORE people for their unique support. We also thank E. Campioni and M. Peters for their extremely useful comments on a previous draft of this work. 


\section{Introduction}

When considering common agency games with complete information, it is well know that the arbitrary restriction to simple take-it or leave-it offers for principals implies a loss of generality. Recent researches developed several ways to characterize through simple and tractable mechanisms the set of equilibria of games where multiple principals are competing in the presence of a single agent. ${ }^{1}$

In a recent paper, Peters [3] identifies a set of restrictions on players' preferences, denoted "no-externality conditions", under which one can safely restrict principals strategies to be take-it or leave-it offers. Interestingly, these conditions are satisfied by a large class of common agency games. ${ }^{2}$

The present note argues that these conditions are in general not sufficient when the agent takes some non contractible actions. We construct an example of a complete information common agency game which satisfies the no-externality conditions as stated by Peters. In this game, at least one equilibrium implementable through menus, cannot be sustained take-it or leave-it offers, which contradicts Theorem 3 and 4 in Peters [3]. The example is constructed in a moral hazard scenario and uses an intuition similar to that suggested in Peters [4].

\section{The model and the counterexample}

We present here a simplified version of the general structure for common agency games as it was originally developed in Peters [2]. In the particular case where no incomplete information is considered, and the agent is only taking a non-contractible action, our framework coincides with that of Peters [3].

We refer to a scenario where there are a number of principals (indexed by $j \in N=\{1, \ldots, n\}$ ) contracting with one agent (denoted by index, 0 ). We assume that the agent takes an effort $e$ from the set $E$ and obtains an allocation $y_{j} \in Y_{j}$ from principal $j$. The effort choice is not contractible.

The payoff to principal $j \in\{1, \ldots n\}$ is represented by the von Neumann-Morgenstern utility function $v_{j}: \times_{k \in N} Y_{k} \times E \rightarrow \mathbb{R}_{+}$. For the agent the payoff is represented by the function $u$ : $\times_{k \in N} Y_{k} \times E \rightarrow \mathbb{R}_{+}$.

Principals compete by simultaneously offering menus. A menu for principal $j$, say $\gamma_{j}$, is a subset of the set of feasible offers $Y_{j}$. The agent chooses an item $y_{j} \in \gamma_{j}$ from each principal $j$ and a non contractible effort $e \in E$.

\footnotetext{
${ }^{1}$ In two influential works, Peters [2] and Martimort and Stole [1] show that there no loss of generality is in fact involved in restricting principals to the use of menus over the relevant alternatives. In other words, menus can efficiently replace any sort of indirect communication mechanism (this is usually referred to as the Delegation Principle).

${ }^{2}$ Peters [3] provides several examples.
} 
We assume that all the relevant spaces satisfy standard regularity assumptions (for a general discussion, the reader can refer to Peters [2] and Peters [3]). We focus our attention on the pure strategy Subgame Perfect Equilibria (SPE) of the game.

Theorem 3 and 4 in Peters [3] identify a set of sufficient conditions on players preferences guaranteeing that every (pure strategy) equilibrium outcome of a game where principals are allowed to use menus can be supported as a pure strategy equilibrium in a simpler game where principals strategies are restricted to be take-it or leave-it offers. In the simple moral hazard framework that we are introducing here, these conditions can be stated in the following way:

For every principal $j \in N$ :

A. There exists a function $V_{j}: Y_{j} \times E \rightarrow \mathbb{R}_{+}$such that for all $\left(y_{1}, y_{2}, \ldots, y_{n}\right) \in Y$, and for all $e \in E$

$$
v_{j}\left(y_{1}, y_{2}, \ldots, y_{n}, e\right)=V_{j}\left(y_{j}, e\right)
$$

B. For any closed subset $B \subset Y_{j}$ there is a $y_{j} \in B$ such that

$$
u\left(y_{j}, y_{-j}, e\right) \geq u\left(y_{j}^{\prime}, y_{-j}, e\right)
$$

for all $y_{j}^{\prime} \in B, y_{-j} \in Y_{-j}$, and $e \in E$.

We introduce here a simple example that shows that these two conditions are indeed not sufficient.

We consider a situation where there are two principals (called Principal 1 and Principal 2) and one agent. The scenario is one of pure moral hazard. That is, the agent is not allowed to take any contractible action.

Principal 1 can take the three decisions $a, b$ or $c$. In the same way, Principal 2 can also take the three actions $A, B$ and $C$. The agent can choose her level of effort in the set $\left\{e^{\prime}, e^{\prime \prime}\right\}$. The level of effort is not contractible.

If the agent chooses the level of effort $e^{\prime}$, the corresponding payoffs are given by the following matrix:

\begin{tabular}{|c|ccc|}
\hline & $A$ & $B$ & $C$ \\
\hline$a$ & $(1,1,0)$ & $(1,2,0)$ & $(1,3,0)$ \\
$b$ & $(4,1,1)$ & $(4,2,4)$ & $(4,3,4)$ \\
$c$ & $(3,1,4)$ & $(3,2,4)$ & $(3,3,4)$ \\
\hline
\end{tabular}

where the first element in each cell refers to the payoff of Principal 1, the second one to the payoff of Principal 2 and the last one to that of the agent. Payoffs associated to the choice of $e^{\prime \prime}$ are:

\begin{tabular}{|c|ccc|}
\hline & $A$ & $B$ & $C$ \\
\hline$a$ & $(0,3,1)$ & $(0,1,1)$ & $(0,0,1)$ \\
$b$ & $(1,3,2)$ & $(1,1,2)$ & $(1,0,4)$ \\
$c$ & $(2,3,4)$ & $(2,1,4)$ & $(2,0,4)$ \\
\hline
\end{tabular}


It is easy to show that this game fulfills conditions (A) and (B). First, the agent has a weak preference ordering over the actions of each principal that is independent of her effort choice and of the other principal's actions. Second, each principal's payoff is only affected by his own action and by the agent's effort.

Following Peters, we restrict our attention to take-it or leave-it offers. Allocations are determined in the following way: each principal simultaneously and independently announces a decision. Given the announcements, the agent chooses her effort level and payoffs are implemented.

We now write down the whole set of continuation payoffs for each array of principals' decisions, taking into account the optimal effort choice of the agent:

\begin{tabular}{|c||c|c|c|}
\hline & $A$ & $B$ & $C$ \\
\hline \hline$a$ & $e=e^{\prime \prime}$ and $(0,3,1)$ & $e=e^{\prime \prime}$ and $(0,1,1)$ & $e=e^{\prime \prime}$ and $(0,0,1)$ \\
\hline \multirow{2}{*}{$b$} & $e=e^{\prime \prime}$ and $(1,3,2)$ & $e=e^{\prime}$ and $(4,2,4)$ & $\begin{array}{l}e=e^{\prime} \text { and }(4,3,4) \\
e=e^{\prime \prime} \text { and }(1,0,4)\end{array}$ \\
\hline \multirow{2}{*}{$c$} & $e=e^{\prime}$ and $(3,1,4)$ & $e=e^{\prime}$ and $(3,2,4)$ & $e=e^{\prime}$ and $(3,3,4)$ \\
& $e=e^{\prime \prime}$ and $(2,3,4)$ & $e=e^{\prime \prime}$ and $(2,1,4)$ & $e=e^{\prime \prime}$ and $(2,0,4)$ \\
\hline
\end{tabular}

One should note that the agent's optimal choice is not always unique.

The outcome $(4,2,4)$ cannot be sustained as an equilibrium if principals are restricted to the use of take-it or leave-it offers mechanisms. For every possible continuation game, if Principal 1 plays $b$, then Principal 2 will always prefer to play $A$ rather than $B$.

We will now show that the outcome $(4,2,4)$ can indeed be supported at equilibrium if principals are allowed to offer menus over the relevant alternatives.

Let us consider the payoff matrix:

\begin{tabular}{|c|ccc|}
\hline & $A$ & $B$ & $C$ \\
\hline$a$ & $(0,3,1)$ & $(0,1,1)$ & $(0,0,1)$ \\
$b$ & $(1,3,2)$ & $(4,2,4)$ & $(1,0,4)$ \\
$c$ & $(3,1,4)$ & $(2,1,4)$ & $(2,0,4)$ \\
\hline
\end{tabular}

that corresponds to the agent's choice of $e^{\prime \prime}$ when she is offered $(b, C),(c, C)$ or $(c, B)$, and with the choice of $e^{\prime}$ when $(c, A)$ is proposed. This corresponds to one of the possible continuation games associated to the effort choice of the agent.

Consider the strategies consisting in Principal 1 offering the menu $\{b, c\}$ and Principal 2 offering the degenerate menu $\{B\}$. A best reply for the agent is to pick the element $\{b\}$ from the first menu and exert the effort $e^{\prime}$. As a consequence, the outcome $(4,2,4)$ is implemented.

Let us show that no principal has a unilateral deviation. First, Principal 1 has no interest in deviating, since he is already at his maximal payoff.

Principal 2 has no reason to deviate either. If he played $\{C\}$, he would get a payoff of 0 , irrespectively of what the agent chooses from Principal 1's menu and provided that she is playing 
the effort $e^{\prime \prime}$. If Principal 2 played $\{A\}$, the agent would choose $c$ from Principal 1's menu, making the deviation not profitable. Hence, there is no profitable deviation using degenerate menus. It follows that the deviation to the menu $\{A, C\}$ as well as all deviations combining $\{B\}$ with $\{A\}$ and/or $\{C\}$ will not be profitable either.

One should notice that Peters [4] finds a similar result, in the context of multi-principal multi-agent games . He introduces an example showing that, in this environment, the "noexternality conditions" are not sufficient to recover the Revelation Principle. His example relies on existence of multiple equilibria in the effort game played among agents. Our example relies on the same kind of argument, constructing a game where a single agent is indifferent between two allocations.

\section{Discussion}

The counterexample shows that the published version of Theorem 3 in Peters [3] is not valid for environments with moral hazard. ${ }^{3}$ The problem in the proof appears when considering unilateral deviations in the take-it or leave-it offer game. In this regard, Peters states: "It is possible that in the original game, the agent's choice from the menus of the other principals might not coincide with $\widetilde{\gamma}_{-j}\left(\hat{e}^{\prime}\right)$ [the non-deviating principals' strategies in the candidate to equilibrium with take-it or leave-it offers] but by part ( $i$ ) of the no-externalities condition [the fact that the payoff of each principal is independent of the other principals' actions], this would not affect the payoff to the deviating principal". ${ }^{4}$ This statement is supported by the claim that the response of the agent (her effort choice) to a deviation of one principal will be the same in the game with menus and in the one with simple take-it or leave-it offers. In the counterexample, even if it is true that the same level of effort is still optimal, there are continuation games where other effort levels are optimal as well.

\section{References}

[1] David Martimort and Lars A. Stole. The revelation and delegation principles in common agency games. Econometrica, 70(4):1659-1673, July 2002.

[2] Michael Peters. Common agency and the revelation principle. Econometrica, 69(5):13491372, September 2001.

[3] Michael Peters. Negociation and take-it or leave-it in common agency. Journal of Economic Theory, 111(1):88-109, July 2003.

[4] Michael Peters. Pure strategy and no-externalities with multiple agents. Economic Theory, 23:183-194, 2004.

\footnotetext{
${ }^{3}$ Since Theorem 4 in Peters [3] is a general version of Theorem 3, it will also fail to apply in moral hazard scenarios.

${ }^{4}$ Peters [3], p. 100.
} 